\title{
Lifetime-enhancement Schemes for Power-constrained Routing in Wireless Sensor Networks: A Survey
}

\author{
G. Pius Agbulu ${ }^{1}$, G. Joselin Retna Kumar ${ }^{2}$ \\ $1^{*}$ Acces Research Lab, Electronics and Instrumentation Engineering (EIE) \\ SRM Institute of Science and Technology, Chennai-603 203, \\ Tamil Nadu, India, gpagbulu@ gmail.com \\ ${ }^{2}$ Department of Electronics and Instrumentation Engineering (EIE) \\ SRM Institute of Science and Technology, Chennai-603 203, \\ Tamil Nadu, India, joselinr@ srmist.edu.in
}

\begin{abstract}
Notwithstanding the broad applicability of wireless sensor network (WSN), the successful development and deployment in various real-world applications is currently challenging. The node-devices are powered by constrained-batteries that are typically impossible to substitute after deployments. Therefore, WSNs are energy-constrained and liable to early failure while expected to run for long-terms without battery replenishments. Diverse researchers have recently presented specific lifetime enhancement routing solutions based on data aggregation technology targeted at reducing energy-consumption, and improving the communication reliability of various WSNs. This paper presents a brief survey of the research field of data aggregation-ware routing in energy-constrained WSN. The paper overviews and classifies various existing routing solutions based on their delivery models and also identifies some of their major drawbacks.
\end{abstract}

Key words WSNs; Aggregation; Lifetime, Communication; Batteries; Energy consumption; Routing.

\section{INTRODUCTION}

A wireless sensor network is formed using assemblages of low-cost node devices that embed sensors, transceivers, processors, and storage modules. Some applications include smart city, smart surveillance, smart farming, and air-quality (AQ) monitoring [1], [2]. Figure 1 shows an architecture of WSN. In several WSN applications, massive numbers of node devices are deployed in harsh neighborhoods to observe specific physical phenomena and transfer the recorded sensory data to a remote gateway [3]. Characteristically, this makes it almost impossible and highly-priced to alternate the batteries of the nodes upon depletion. Thus, the networks are energy-constrained and failure inclined while expected to run for an extended period without battery substitutions [4].
Because of the restrained energy-resource and failure prone dispositions of the network, the design of lifetime-enhancement and aggregation-aware routing solutions in WSN yet continues to be a challenging and significant research field in recent years[5],[6],[7],[8].With data aggregation, identical sensory packets can be amassed at some nodes and conveyed to the BS in single or multi-hop fashion. Notably, the strategy of aggregating similar data curtails the network's traffic, reduces collision overheads, and promotes low energy consumption at the nodes [7].

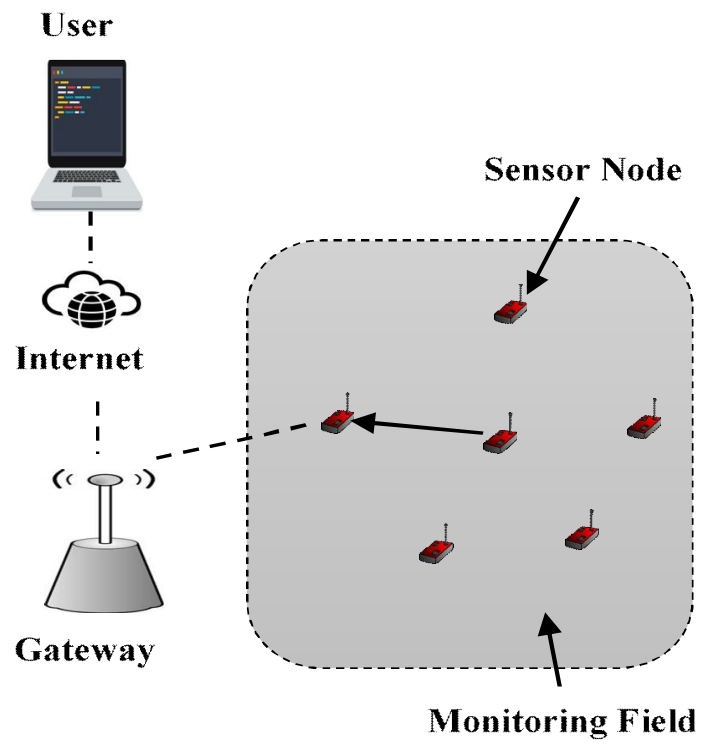

Figure 1: An architecture of WSN

The design of routing scheme and network design are key factors that impact the aggregation performance. In over-all, aggregation techniques in WSN are categorized into structured and structure-less techniques. Structured aggregation exploits precise architecture in implementing data aggregation. Meanwhile, data aggregation with structure-less techniques does not utilize particular architecture. Structured architectures are generally categorized into hierarchical and flat networks. In flat network structures, every node characteristically serve 
identical role and implement sensing operations collaboratively. Hierarchical network structures exploit clustering technology, reservation-based scheduling, and node heterogeneity [8], [9], 10]. Typically, the hierarchical structures are categorized into chain and clustered architectures.

Routing plays a vital role in WSN configuration by defining the optimal communication routes for data transfer from the nodes to the gateway. Explicitly, the model of routing for any given sensor network impacts the lifetime of the nodes Therefore many researchers have made significant efforts to propose various solutions to deal with the routing challenges of various WSN recently, [13],[14],[15].This paper presents a brief survey of the research area of lifetime enhancement routing in energy-constrained WSN. The paper classifies various current routing solutions based on their communication and identifies the fundamental drawbacks of some of the solutions.

The remaining portion of this paper is arranged as follows. Section 2 presents a detailed review of lifetime enhancement aggregation-ware routing solutions in WSNs and identifies the challenges the fundamental drawbacks of some of the solutions. Finally, Section 3 concludes this work.

\section{REVIEW OF LIFETIME-ENHANCEMENT ROUTING PROTOCOLS IN WSN}

At recent years, diverse researchers have presented specific data aggregation-aided routing design solutions for WSNs aiming at encouraging node lifetime enhancements. Depending on their modes of sensing and communication, they can be categorized into time-driven, query-driven, and event-driven routing categories. Figure 2 shows some suitable applications for each routing model category based on the monitoring operation needs. Here, we review several existing standard time-driven, event-driven, and query-driven data aggregation-aided routing schemes in WSN.

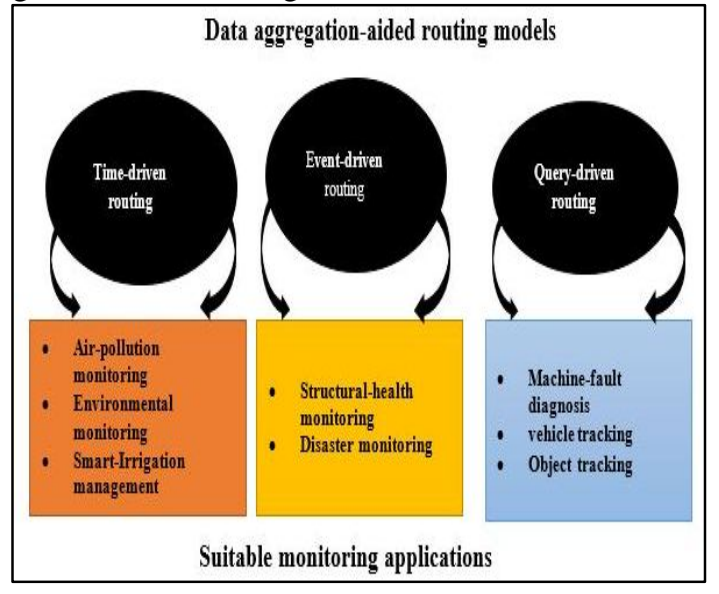

Figure 2: Classification of WSN routing solutions and suitable applications

\subsection{Time-driven Routing Solutions}

The time-driven design is proper for WSN applications requiring periodic data observations. In that view, sensor nodes intermittently turn on their sensing and transmitting components, observe their vicinities and disseminate the data of interest periodically. Numerous researcher have recently proposed diverse WSN time-driven routing solutions. Most of these solutions are principally dedicated to use of hierarchical routing methods wherein the nodes are divided into clusters. The aims in cluster-based WSNs is to split the nodes into some cells of clusters such that every cell can have a head node called cluster head $(\mathrm{CH})$ that periodically aggregates and communicate the sensed observations of the members.

The low energy adaptive clustering hierarchy (LEACH) is a classical time-driven cluster-based protocol and one of most famous routing solution in WSN [16]. LEACH is realized in two phases: The first stage is the setup-phase wherein $\mathrm{CHs}$ are assigned and introduced to the cell members. The second-stage is called the steady phase. In this stage, the nodes broadcast their data to their cell heads based on TDMA fixed slots. Though LEACH has some deficiencies like poor allocations of $\mathrm{CH}$; over the past, a lot of developments have been done on LEACH protocol by diverse researchers [30-33]. The LEACH-C (LEACH-centralized protocol) is an upgraded form of LEACH [17]. LEACH-C is intended to optimize the $\mathrm{CH}$ distribution in $\mathrm{LEACH}$ with a centralized control strategy. In LEACH-C protocol, the BS gauges the node's average residual energy throughout the system and solely permits the node devices possessing residual energy proportionate to the gauged average node's residual energy to implement functionalities of $\mathrm{CH}$ per communication round.

The LEACH-F (LEACH with fixed clusters) [18], is as well, an improved centralized form of LEACH protocol. In LEACH-F, setup phases are not needed at every round. Instead, the $\mathrm{CH}$ roles are alternated amongst the various nodes. The DF-LEACH (data fusion oriented clustered routing protocol based on $\mathrm{LEACH}$ ) is proposed in [19] lately to promote both low energy-usage at the node devices and ensure prolonged survival of the network. This enhanced model named DF-LEACH realizes data fusion at the heads in a hop-by-hop fashion before eventually delivering the observations to the base station.

Abdusalam et al. in [20] proposed a periodic WSN-based air quality data gathering method based on LEACH-aggregation strategy. However, this scheme has some limitations like crowded center-effects from relay and aggregator roles imposed on the cluster heads. Also, it has high probabilities of poor $\mathrm{CH}$ node management due to the inherent drawbacks in LEACH. Moreover, the proposed scheme may not be suitable for time- driven outdoor pollution monitoring with broad coverage requirements. The hybrid energy-efficient distributed clustering protocol (HEED) [21], is another popular time-driven hierarchical 
G. Pius Agbulu et al., International Journal of Emerging Trends in Engineering Research, 9(2), February 2021, 140 - 147

Table 1: Analysis of Time-driven routing solutions

\begin{tabular}{|c|c|c|c|}
\hline S.No & Author & Remarks & Gaps Identified \\
\hline 1 & Ahmed et al. [29]. & $\begin{array}{l}\text { SEP-CS: This protocol } \\
\text { defines three tiers of } \\
\text { heterogeneity. } \\
\text { Applies compressive sensing } \\
\text { (CS) at the nodes to achieve } \\
\text { reduced propagation costs. }\end{array}$ & $\begin{array}{l}\text { High packet loss probability. } \\
\text { Factors of connectivity and } \\
\text { coverage at the compressing } \\
\text { node-devices are not } \\
\text { considered. }\end{array}$ \\
\hline 2 & Tarhani et al. [26]. & $\begin{array}{l}\text { SEACH: It applies } \\
\text { cluster-based periodic } \\
\text { communication model with } \\
\text { dedicated relay cooperative } \\
\text { communication. }\end{array}$ & $\begin{array}{l}\text { - Reduced delivery ratio since } \\
\text { optimal relay distribution is not } \\
\text { guaranteed. } \\
\text { - Relay node coverage are node } \\
\text { secured. }\end{array}$ \\
\hline 3 & Mehmood et al. [22]. & $\begin{array}{l}\text { - SeLeZoR: The node devices are } \\
\text { divided into zones of clusters. } \\
\text { - The zone-head sends the data to } \\
\text { the base station using a secure } \\
\text { and energy efficient } \\
\text { mechanism. }\end{array}$ & $\begin{array}{l}\text { - The proposed solution is } \\
\text { confined to small scale } \\
\text { monitoring operation. } \\
\text { - The MAC protocol exploited by } \\
\text { the nodes is not detailed. }\end{array}$ \\
\hline 4 & Abdusalam et al. [20]. & $\begin{array}{l}\text { - LEACH routing protocol is } \\
\text { adapted for WSN based Air } \\
\text { Quality data aggregation. }\end{array}$ & $\begin{array}{l}\text { - Reduced lifespan of } \mathrm{CH} \text { nodes } \\
\text { from relaying and aggregation } \\
\text { duties. } \\
\text { - Overheads of poor } \mathrm{CH} \text { node } \\
\text { distributions. } \\
\text { - Not suitable for outdoor } \\
\text { pollution monitoring with wide } \\
\text { coverage demands. }\end{array}$ \\
\hline 5 & Zhansheng et al. [27]. & $\begin{array}{l}\text { - GRMRP: It initially splits the } \\
\text { monitoring space into some } \\
\text { cluster grids. } \\
\text { - Only a node is kept active whilst } \\
\text { other sensor transit to sleep. }\end{array}$ & $\begin{array}{l}\text { - More energy is expended due to } \\
\text { complex rules in route set-up. }\end{array}$ \\
\hline
\end{tabular}

routing solution based on node clustering. In HEED, the initial chances of nodes performing cluster head roles depends on their proximities to adjoining nodes and residual battery-powers. Reference [22] presents a time-driven zone-based routing protocol named SeLeZoR. In SeLeZoR, the nodes are divided into zones to monitor various pollution parameters in their neighborhoods periodically. The zone-head sends the data to end base station exploiting an energy-efficient and secure technique. The KDUCR protocol (K-means and Dijkstra algorithm-based routing) [23], is a time-driven data aggregation-aided routing model that 
employs advantages of Dijkstra shortest route algorithm and K-means learning-based clustering algorithm. The central notion in WSN K-means clustering strategy is to arbitrarily choose K-points in the sensing field and designate the sensor node devices to their closest points to build K-regions of clusters across the area. The learning algorithm estimates each K-cell's centroid repeatedly till the points converge. This method decreases the complexities correlated with establishing clusters at distinct layers in WSNs. The K-means with LEACH (KM-LEACH) protocol presented in [24] organizes the sensor nodes into clusters by the K-means method and uses LEACH protocol for $\mathrm{CH}$ election. Kulaib et al. in [25] recommended a time-driven enhancement on dynamic voting-hop (DV-hop) scheme based on K-means. The suggested solution employs DV-hop algorithm to compute the hops between a sensor node and a distant anchor node.

In [26], Tarhani et al. propose a classical time-driven scheme called the scalable energy-efficient clustering hierarchical routing protocol (SEACH). SEACH considers dedicated groups of relay nodes to serve the cluster heads transmit their aggregated observations to the destination, which results in prolonged life-spans of the cluster heads and better scalability. Reference [27] introduces a time-driven scheme called grid-based reliable multi-hop routing protocol (GRMRP).GRMRP initially splits the monitoring space into some cluster grids. The grids are eventually sectioned into specific cover sectors. Only a node is kept active whilst other sensor transit to sleep.

The routing models reviewed above are designed for time-driven static-homogeneous WSNs set-ups. Mainly, clustered WSN structures can be either homogeneous or heterogeneous. In a homogenous configuration, the nodes possess similar initial energy, while in heterogeneous set-ups; the nodes have unlike initial energies. Stable election protocol (SEP) is one of the well-known periodic WSN heterogeneous routing scheme based clustering [28]. SEP defines two tiers of heterogeneity that differentiates the nodes into advanced node (AN), and normal node (NN) categories. The advanced nodes are regarded to possess extra energy more than the normal nodes. In [29], Ahmed et al. recommend an adapted variant of SEP protocol based on compressive sensing (CS) named SEP-CS .This protocol defines three tiers of heterogeneity and applies compressive sensing (CS) at the nodes to achieve reduced propagation costs. Table 1 gives an overview of some of the works overviewed here and their identified limitations.

\subsection{Event-driven Routing Solutions}

In event-driven WSN formation, sensors respond swiftly to variances in values of observed sensory attributes corresponding to a pre-defined event. Some standard WSN event-driven routing solutions proposed by various researchers lately are surveyed here.
Manjeshwar and Agarwal in [30] proposed the threshold sensitive energy-efficient (TEEN) protocol. TEEN is an event critical cluster-based data gathering scheme that uses a soft and hard thresholding scheme to reduce the data propagation cost. The hard threshold bound allows the nodes only to disseminate their observation if they relate to attributes of interest events. Concurrently, the soft limit sanctions the nodes to omit data propagations in trivial or no changes in their perceived observations. The event-driven clustering (EDC) protocol is another energy-efficient clustered -based routing solution introduced in [31] .In, EDC the clustering stage of the nodes is actuated by events episodes in the network. EDC exploits MAC-based synchronous techniques to coordinate the data transmissions between the $\mathrm{CH}$ nodes and their members.

In [32], Roy and Das present the cluster-based event-driven routing protocol (CERP). In this scheme named CERP, the sensor nodes build clusters employing similar event-based grouping strategy based on episodes of events in the sensing zone. In the formation stage, all nodes calculate a competing cost with regards to the demanded energy for aggregation and communication to define their respect heads. In [33], Razzaq et al. present an even-based K-means based clustering scheme, which considers a fixed-packet length and weight functions in making $\mathrm{CH}$ election decisions. Furthermore, Wang et al. in [34] proposed the energy-efficient compressive sensing-based clustering routing (EECSR) protocol to resolve hitches of hotspot and power consumption in clustered event-driven WSNs. Table 2 gives an overview of some of the works overviewed above and their identified limitations.

\subsection{Query-driven Routing Solutions}

In query-driven WSN, the sensor nodes perform sensing and data delivery on-demand based on interest messages or queries received from the base station (BS). Specifically, the interest messages may indicate a set of the duties allotted to the node devices by the BS, like object or event tracking. Mainly, both event-driven and query-driven models are well suited for time-critical WSN applications.

In [35], Schurgers et al. suggested the gradient-based routing (GBR) as a query-based distributed multi-hop data gathering strategy and improved modification of directed diffusion (DD). GBR is proposed to reinforce the sensors in determining least hops to the sink from their adjacent neighbors. Thus, GBR enables each node to estimate hops from its neighbor are called as 'height,' employing interest or queries circulated by the sink. Most related GBR schemes have been established in the various recent studies to reinforce extended WSN lifetime and energy-saving capacity. Reference [36] recommends the network coding based GBR named GBR-NC. It merges generic-GBR with random linear network coding technology. GBR-NC is proposed to curtail the outlays of energy tax and interest replication in GBR. It 
G. Pius Agbulu et al., International Journal of Emerging Trends in Engineering Research, 9(2), February 2021, 140 - 147

relies on MAC-based feedbacks to tackle overheads of pack loss from network-coding activities. suggested a gradient-based multi-hop routing strategy to support reduced energy tax in sensor networks.

Table 2: Analysis of Event-driven routing solutions

\begin{tabular}{|c|c|c|c|}
\hline S.No & Author & Remarks & Gaps Identified \\
\hline 1 & $\begin{array}{l}\text { Manjeshwar and } \\
\text { Agarwal [30]. }\end{array}$ & $\begin{array}{l}\text { - TEEN: It uses two limits, a soft, } \\
\text { and a hard threshold to } \\
\text { coordinate the communication } \\
\text { phase of the network. }\end{array}$ & $\begin{array}{l}\text { - Poor } \mathrm{CH} \text { node distribution at } \\
\text { muti-level. } \\
\text { - High chances of } \mathrm{CH} \text { node } \\
\text { isolation across. } \\
\text { - More energy is dissipated from } \\
\text { incessant sensing operations. }\end{array}$ \\
\hline 2 & Wang et al. [34]. & $\begin{array}{l}\text { EECSR:It merges cluster-based } \\
\text { Event-driven communication } \\
\text { model with compressive } \\
\text { sensing mechanisms. }\end{array}$ & $\begin{array}{l}\text { - Control packet flooding } \\
\text { overheads during position } \\
\text { and energy information } \\
\text { exchange at set-up phase. } \\
\text { - Overheads of reduced CH } \\
\text { lifetimes from imposed roles } \\
\text { of relays and aggregators. }\end{array}$ \\
\hline 3 & Roy S and Das [32]. & $\begin{array}{l}\text { - CERP: The nodes form clusters } \\
\text { based on events in the } \\
\text { monitoring field. } \\
\text { - CH nodes selection is based on a } \\
\text { transceiving and aggregation } \\
\text { energy metrics. }\end{array}$ & $\begin{array}{l}\text { - Poor cluster distribution at } \\
\text { set-up phase. } \\
\text { - Crowded center issues from } \\
\mathrm{CH} \text { isolation. }\end{array}$ \\
\hline 4 & Zeng-Wei et al. [31]. & $\begin{array}{l}\text { - In, EDC clustering is actuated } \\
\text { by events episodes. } \\
\text { - The steady phase is coordinated } \\
\text { using MAC-based technique. }\end{array}$ & $\begin{array}{l}\text { High packet-loss rate from } \\
\text { energy-hole overheads. } \\
\text { - Increased energy dissipation } \\
\text { from poor cluster distribution } \\
\text { at event episodes. }\end{array}$ \\
\hline
\end{tabular}

Reference [37] proposes a multi-sink GBR scheme to encourage enhanced coding actions in diverse on-demand sensor networks, and also aid lessened signaling issues associated with coding scenarios. This scheme systematically assesses the absolute quantity of nodes in the sensing sector to enforce coding processes and utilize multi- sinks addresses to convey NC-coded packets. This scheme has no serious issues; however, in multi-sink structures, sink positions can affect global transmission performances Quang et al. in [38]
This strategy employs two-hop knowledge to make route choices. In [39], Ren et al. propose a power-dynamic routing policy named ADA (attribute-aware data aggregation). This scheme incorporates a timing algorithm with advanced dynamic routing model . Limin et al. in [30] introduced the FSG protocol (fine-grain gradient sinking). FSG establishes safe routes for data transmission by aiding the BS to translate the complete hop information into gradient data. Migabo et al. in [41] recommend the CB-GBR (competing energy 
G. Pius Agbulu et al., International Journal of Emerging Trends in Engineering Research, 9(2), February 2021, 140 - 147

balancing gradient-based routing) protocol. CB-GBR is and obstacles, issues in practical query-based sensor recommended networks.

Table 3: Analysis of Query-driven routing solutions

\begin{tabular}{|c|c|c|c|}
\hline S.No & Author & Remarks & Gaps Identified \\
\hline 1 & $\begin{array}{c}\text { Schurgers et } \\
\text { al.[35]. }\end{array}$ & $\begin{array}{l}\text { - GBR: It improves the } \\
\text { Diffusion (DD) protocol. } \\
\text { - Shortest path computation } \\
\text { with 'Height' parameter. }\end{array}$ & $\begin{array}{l}\text { - High latency from single path approach. } \\
\text { - High energy consumption from arbitrary } \\
\text { interest message diffusion. }\end{array}$ \\
\hline 2 & $\begin{array}{l}\text { Miao et al. } \\
{[36] .}\end{array}$ & $\begin{array}{l}\text { - GBR-NC: It unifies random } \\
\text { linear network-coding } \\
\text { (RLNC) technology with } \\
\text { Generic-GBR model. }\end{array}$ & $\begin{array}{l}\text { - Relies on a random traditional feedback, } \\
\text { which can be negatively affected by drifts in } \\
\text { connectivity at the coding-channels. }\end{array}$ \\
\hline 3 & $\begin{array}{c}\text { Kennan et al. } \\
\text { [44]. }\end{array}$ & $\begin{array}{l}\text { - EEOGRP: It combines } \\
\text { Generic-GBR with OMLRP } \\
\text { (on-demand-multi-hop } \\
\text { information based on } \\
\text { routing protocol. }\end{array}$ & $\begin{array}{l}\text { - The proposed solution is confined to small } \\
\text { scale on-demand monitoring operation. } \\
\text { - The MAC-protocol exploited by the nodes is } \\
\text { not detailed. }\end{array}$ \\
\hline 4 & Hao et al. [42]. & $\begin{array}{l}\text { - GMPR: It combines low-duty } \\
\text { cycling mechanisms with } \\
\text { generic-GBR. }\end{array}$ & $\begin{array}{l}\text { - Not suitable of query-based monitoring with } \\
\text { periodic event flow. } \\
\text { - Increased energy dissipation from random } \\
\text { Interest message diffusion. } \\
\text { - Single path overheads. }\end{array}$ \\
\hline 5 & Qiu et al.[45] & $\begin{array}{l}\text { - MGRP: It utilizes the nodes' } \\
\text { routing information to } \\
\text { evaluate the distance between } \\
\text { nodes in interest vicinities. }\end{array}$ & $\begin{array}{l}\text { - Delayed response due to intricate rules in hop } \\
\text { computation. } \\
\text { - Increased energy dissipation from arbitrary } \\
\text { interest message diffusion among sensor } \\
\text { nodes. }\end{array}$ \\
\hline
\end{tabular}

as an enhanced design of competing-GBR. It utilizes identical concepts of GBR-C but employs multiple BS as opposed to GBR-C that considers a single BS. Hao et al. in [42] suggested the GMPR (Gradient-based multipath routing protocol), which is targeted at reinforcing decreased node failures and improved transmission performances through duty-cycling. It applies the sensory reports to gauge the distances of neighboring nodes. Liu et al. [43] explored various scenarios of building gradient information fields in query-driven WSNs and recommended two GBR-based solutions to deal with task-preferences, energy dissipation
In [44], Kannan et al. introduce an energy-efficient optimal GBR protocol named as EEOGRP. This modified GBR also consolidates the advantages of on-demand-multi-hop information routing (OMLRP). EEOGRP is fully

query-driven and intends to meet the essentials of the enhanced route selections and better energy-saving in on-demand WSNs. Moreover, Qui et al. [45] recommended the MGRP (multi-gradient routing protocol), which is meant to aid curtailed traffics and energy outlays in on-demand sensor networks. Table 3 gives an overview of some of the works overviewed and their identified limitations. 
G. Pius Agbulu et al., International Journal of Emerging Trends in Engineering Research, 9(2), February 2021, 140 - 147

\section{CONCLUSION}

Despite their full capabilities, the successful development and deployment of WSNs in various real-world operations are currently challenging. The node devices are powered by constrained-batteries that are mostly impractical to substitute after the system deployment. Moreover, conventional sensor networks produce sizeable amounts of redundant data. Conveying such vast quantities of readings from the nodes to the central destination can explicitly cause the sensors to consume their inadequate energy-reserves faster and fail prematurely. Given that sensors in local neighborhoods may also observe similar events, it is ineffectual to convey the recorded observations directly to the destination. Thus, it becomes imperative to consolidate the node's observations into well-valuable data at intermediate sensor nodes with data aggregation technology to assure prolonged lifetime of the nodes and reduced overheads of redundancies In this paper, we have briefly reviewed and classified various lifetime enhancement routing solutions for energy-constrained WSNs based on their communication models and also highlighted some of the fundamental drawbacks.

\section{REFERENCES}

1. G. Elumalai, R. Ramakrishnan. A Novel Approach to Monitor and Maintain Database About Physiological Parameters of (Javelin) Athletes Using Internet of Things (IoT), Wireless Pers. Commun., vol. no. 111,pp. 343-355,2020.

2. M.Shafiq, A. Humaira,U. Ata, and T. Shireen. Systematic Literature Review on Energy Efficient Routing Schemes in WSN - A Survey, Mobile Networks and Applications, vol. 3, 2020

3. P. Lavanya, and R. Sudha. A Study on WSN Based IoT Application in Agriculture, in Proc. IEEE ICCES, Coimbatore, India, pp.1046-1054, 2018.

4. M.B, Mollah, S. Zeadally, and M.K. Azad, M.K. Emerging Wireless Technologies for Internet of Things Applications: Opportunities and Challenges, Springer, Cham, 2020.

5. P.G. Agbulu , G.R.-K. Joselin , A. Vimala Juliet . AERAA-DAAR: Algorithm for energy-resourceful attribute-aware data aggregation and routing in wireless sensor networks. Ad Hoc Sens.Wirel. Ne vol 46 no.3-4,pp.265-296,2020.

6. W. J. Jiayao, O .Timothy, L. Tawose Jiang, and Z. Zhao.A New Data Fusion Algorithm for Wireless Sensor Networks Inspired by Hesitant Fuzzy Entropy, Sensors, vol.19, no.4, pp.784,2018.

7. K.L., Joshitha, and S. Jayashri. Performance analysis for lifetime improvement of a regression based clustered network through cluster member allocation and secondary cluster head selection in a distributed WSN, Cluster. Comput.vol.22, pp.9805-9814, 2019.
8. S. Patnaik, X. Li, and Y.M. Yang. Recent Development in Wireless Sensor and Ad-hoc Networks. Springer, 2014.

9. J.M Kahn,R.H. Katz, and K.S.J Pister. Next Century Challenges: Mobile Networking for Smart Dust, in Proc. 5th annual ACM/IEEE International Conference on Mobile Computing and Networking (MobiCom'99), pp.271-278, 1999.

10. Chugha, A., and Supriya, P. Strengthening Clustering Through Relay Nodes in Sensor Networks, Procedia Computer Science, 132, 689-695, 2018.

11. A. A. Sayyid, H. Harb, M. Ruiz and L. Velasco. ZIZO: A Zoom-In Zoom-Out Mechanism for Minimizing Redundancy and Saving Energy in Wireless Sensor Networks, IEEE Sensors Journal, doi: 10.1109/JSEN.2020.3025188.

12. L. Ming, and W. Yanling. Study on multipoint relays selection based on SINR and residual energy aware in wireless multi-hop networks, J. Intell. Fuzzy Syst., vol. 35,no. 2, pp. 1281-1290,2017

13. Shanti, K.L., and Jayashri, S.,“ Performance analysis for lifetime improvement of a regression based clustered network through cluster member allocation and secondary cluster head selection in a distributed WSN, Cluster Comput. 22, pp.9805-9814,2019.

14. H. Lin and W. Chen. An Approximation Algorithm for the Maximum-Lifetime Data Aggregation Tree Problem in Wireless Sensor Networks. IEEE Trans. Wirel. Commun., vol. 16, no. 6, pp. 3787-3798, 2017.

15. P.G. Agbulu , G J-R. Kumar and A.V. Juliet. A Lifetime- enhancing Cooperative Data Gathering and Relaying Algorithm for Cluster-based Wireless Sensor Networks. Int. J. Dis-trib. Sens. N, vol. 16,no. ,2020

16. W.R Heinzelman, A. Chandrakasan, and H. Balakrishnan, Energy-efficient Communication Protocol for Wireless Micro-Sensor Networks, Proc. IEEE Annual Hawaii International Conference on System Sciences, Maui, Hawaii, USA, 2000.

17. W.R Heinzelman, A. Chandrakasan, and H. Balakrishnan, . An application-specific protocol architecture for wireless micro sensor networks, IEEE Trans. Wirel. Commun. vol.1,no, no. 4, 660-670,2002.

18. L. Chen. and B.H. Zhao. Data fusion oriented routing protocol based on leach, J. Beijing Univ Posts and Telecommun 5, 017, 2009.

19. D. Zhixiang, and Q. Bensheng. Three-layered routing protocol for WSN based on LEACH algorithm, Proc. IET Conference on Wireless and Mobile Sensor Network Shanghai, China pp. 12-14 2017.

20. A. M. Abdulsalam, B.A Ali, and A. Alyatama. Air Quality Monitoring Using a LEACH-based Data Aggregation Technique in Wireless Sensor Network, Ad hoc Sens. Wirel. Ne., vol.32 no.3-4, pp. 275-300, 2016. 
G. Pius Agbulu et al., International Journal of Emerging Trends in Engineering Research, 9(2), February 2021, 140 - 147

21. D. Mehta, and S. Saxena. A Comparative Analysis of Energy Efficient Hierarchical Routing Protocols for Wireless Sensor Networks, Proc. 25th ACM Conference on Computer and Communications Security, Toronto, Canada 15-19 October, 2018.

22. O. Younis, and S. Filmy .Heed: a hybrid, energy-efficient, distributed clustering approach for ad hoc sensor networks, IEEE Tran. Mob.Compt., vol. 3,no. 4, pp. 366-379,2004.

23. A. Mehmood, J. Lloret, and S. Sendra .A Secure and Low-Energy Zone-based Wireless Sensor Networks Routing Protocol for Pollution Monitoring, Wireless Communications and Mobile Computing, vol. 16, no. 17, 2869-2883,2016.https://doi.org/10.1002/wcm .2734.

24. M. Bidaki, R. Ghaemi and S. Tabbakh. Towards Energy Efficient k-MEANS Based Clustering Scheme for Wireless Sensor Net-works, Int. J. Grid Dist. Comp., vol. 9, no. 7, pp.265 -276,2016.

25. R. Kulaib, R.M. Shubair, M.A. Al-Qutayri, and J.W.P. $\mathrm{Ng}$. Improved DV -hop localization using node repositioning and clustering, Proc. 2015 International Conference on Communications, Signal Processing, and their Applications (ICCSPA15), 2015.

26. Tarhani, M., Kavian, Y.S., and Siavoshi, S. SEECH: Scalable Energy Efficient Clustering Hierarchy Protocol in Wireless Sensor Networks, IEEE Sens. J., vol. 14, no. 11, pp. 3944-3954,2014.

27. Z. Chen, and H. Shen. A grid-based reliable multi-hop routing protocol for energy-efficient wireless sensor networks, Int. J. Distrib. Sens. N. 14, no. 3,2018.

28. G. Smaragdakis, I. Matta , and A. Bestavros, SEP: A Stable Election Protocol for Clustered heterogeneous wireless Sensor Networks, Proc. IEEE SANPA-04, pp. 251-261, 2004.

29. M.K. Ahmed, and D.M. Omar. SEP-CS: Effective Routing Protocol for Heterogeneous Wireless Sensor Networks, Ad Hoc and Sens. Wire. Ne. vol. 26,no. 1, 211-232,2015.

30. Manjeshwar and Agarwal D.P. TEEN: A routing protocol for enhanced efficiency in wireless sensor networks, Proc. International Parallel and Distributed Processing Symposium. San Francisco, California, pp. 23-27, 2001.

31. Z. Zeng-wei, W. Zhao-hui, and L. Huai-zhong. An event-driven clustering routing algorithm for wireless sensor networks, Proc. IEEE/RSJ International Conference on Intelligent Robots and Systems, Sendai ,pp. 1802-1806, 2004.

32. S. Roy, and A.K Das. Cluster based event driven routing protocol (CERP) for wireless sensor network, Int J . Compt Appl., vol. 88, 0975-8887,2014.

33. M. Razzaq, G. Kwon and S. Shin. Energy efficient Dijkstra-based weighted sum minimization routing protocol for WSN, in Proc. 2018 Third International Conference on Fog and Mobile Edge Computing (FMEC), Barcelona, pp. 246-251,2018. doi: 10.1109/FMEC.2018.8364074
34. Q. Wang, D. Lin, P. Yang, and Z. Zhang. An Energy-Efficient Compressive Sensing-Based Clustering Routing Protocol for WSNs, IEEE Sens. J. vol.19, no. 10, pp.3950-3960,2019.

35. Schurgers, C., and Srivastava, M. Energy efficient routing in wireless sensor networks, Proc. IEEE MILCOM, McLean, VA, USA, 2001.

36. Lusheng Miao, Karim Djouani, Anish Kurien, Guillaume Noel, Network coding and competitive approach for gradient based routing in wireless sensor networks, Ad Hoc Networks, vol. 10,no.6, pp. 990-1008,2012.

37. M.E . Migabo, T.O. Olwal, K. Djouani, and A.M. Kurien. Cooperative and Adaptive Network Coding for Gradient-Based Routing in Wireless Sensor Networks with Multiple Sinks, J. Comp. Netw. Commun., vol.2017, pp.1-10,2017.

38. P.T.A. Quang, and D.S. Kim, D.S. Enhancing Real-Time Delivery of Gradient Routing for Industrial Wireless Sensor Networks, IEEE Trans. Ind. Informat., vol.8, no. 1, pp.61-68,2014.

39. J. Ren, Y. Zhang, T. Wu, T. He, C. Chen and C., Lin. Attribute aware data aggregation using potential-based dynamic routing in wireless sensor networks, IEEE Trans. Parallel Distrib. Syst., vol 24, no. 5, pp.881-892.

40. Z. Limin, D. Hongsong, L. Bin, Xiaowei, and S. Yi. Analysis of Forwarding Mechanisms on Fine-Grain Gradient Sinking Model in WSN. J. Sig. Process. Sys., vol.51,no. 2 , pp.145-159,2007.

41. M. Migabo, K. Djouani, A. Kurien, and T. Olwal. Gradient-based Routing for Energy Consumption Balance in Multiple Sinks-based Wireless Sensor Networks, Procedia Comput. Sci., vol. 63, pp.488-493,2015.

42. J. Hao, Z. Yao, Huang, K. Zhang, B., and C. Li .A gradient based multiple-path routing protocol for low duty-cycled wireless sensor networks, Wirel. Commun. Mob. Com. J., vol. 16,no. 5, pp.538-549.,2014.

43. S. Liu, J. Du, L. Hui, L. Rui, X. Yang, and K. Sha, K. Energy-Efficient Algorithm To Construct The Information Potential Field In WSN, IEEE Sens. J., vol.17, no. 12, pp.3822-3831,2017.

44. K.N. Kannan, and Paramasivan. Development of Energy-Efficient Routing Protocol in Wireless Sensor Networks Using Optimal Gradient Routing with On Demand Neighborhood Information, Int. J. Distrib. Sens. N., vol.10, pp.2 08023,2014.

45. Y. Qiu, S. Li, Z. Li, Y. Zhang, and Z. Yang. Multi-gradient routing protocol for wireless sensor networks, China Commun. J., vol.14, no. 3. pp.118-129 ,2019. 